\title{
Preface (1994)
}

In the preface to the first edition of The Supernova Story I expressed the hope that, along with telling the story of exploding stars, I might give the reader some appreciation of how astronomy was done. Rereading what I had written there, six years later, I realize that doing science was what the book was about in the very first place. Conventional wisdom in science is so fluid that any account of a particular discipline is dated almost as soon as it reaches the bookseller's shelves. But the overall enterprise of science is much more enduring. You may never be able to step twice into the same river, yet you still know it's a river you've stepped into. While some of the facts, figures, and interpretations I cited in 1988 have changed since I wrote them down, I still feel satisfied that this book presents a reliable account of the process of research in one of the most exciting fields of modern astronomy.

Having said that, I am struck with how little has actually changed despite all that has happened. In most respects, Supernova 1987A has developed about as we expected. The overall decline in its light output, the appearance of gamma rays from its radioactive by-products, the interaction of its outrushing debris with the surrounding interstellar gas, have all proceeded pretty much on schedule. Several hundred new supernovae, most of them very faint, have been observed since 1988, and the brightest of the new crop, which appeared in March 1993, caused almost as much excitement as its predecessor. That one, Supernova 1993J, was enough like Supernova 1987A to show us what we got right in the first place, and enough different to lead us into fascinating new realms of inquiry.

In recent years, new telescopes in space and on the ground have 
helped us discover new pulsars and supernova remnants. And a number of clever and diligent theoretical astrophysicists have come up with sparkling new computer models that highlight the fine details of supernova explosions. The skeleton picture of a supernova we had in 1988 now has much more flesh on its bones. But its overall shape is very similar to what it was back then.

For that reason I have resisted the urge to tinker with the original text, and have not endeavored to bring each fact and figure up to date. By the time you read this, science will surely have moved on again. There may be a dozen new pulsars or three hundred faint supernovae not mentioned here, along with a host of novel theories that confirm or deny possibilities I have raised. While I have tried to clarify ambiguous passages and correct outright misstatements of fact, the body of the first edition stands pretty much as it did when I wrote it. It should serve as a trustworthy guide to our knowledge of supernovae at the time of Supernova 1987A.

Still it is fascinating, after some passage of time, to return to the scene of an event and learn what has happened to its principal characters. I have, accordingly, added an epilogue to the original edition, so that the reader can find out what has happened to Sanduleak -69 202 since 1988. I have also included a section on recent advances in supernova research in that chapter and a few additional references in the bibliography so that the reader may follow further developments in the field with an intelligent eye.

The preparation of this new edition benefited from the work of many besides myself. I would particularly like to thank Stan Woosley for his careful reading of the original text and his many helpful suggestions for improvements. Greg Aldering, John Blondin, Alex Filippenko, Janet Mattei, Dick McCray, Mike Richmond, Brian Schmidt, Michael Seeds, and Nick Suntzeff contributed information and data on late-breaking developments. Shawn Baker provided invaluable help in library research. I have also incorporated revisions proposed by a number of readers and reviewers over the years. Finally, I would like to thank Trevor Lipscombe at Princeton University Press, without whose efforts this paperback version would not have been produced.

Laurence A. Marschall

Gettysburg, Pennsylvania 